\title{
Multimodal Plasmonic Hybrids: Efficient and Selective Photocatalysts
}

Yoel Negrín-Montecelo, Xiang-Tian Kong, Lucas V. Besteiro, Enrique Carbó-Argibay, Zhiming M. Wang, Moisés Pérez-Lorenzo, Alexander O. Govorov, * Miguel ComesañaHermo* and Miguel A. Correa-Duarte*

Y. Negrín-Montecelo, L. V. Besteiro, M. Pérez-Lorenzo, M. A. Correa-Duarte CINBIO, Universidade de Vigo, Department of Physical Chemistry, 36310 Vigo, Spain

E-mail: macorrea@uvigo.es

Y. Negrín-Montecelo, L. V. Besteiro, M. Pérez-Lorenzo, M. A. Correa-Duarte Galicia Sur Health Research Institute (IISGS), CIBERSAM, 36310 Vigo, Spain

X.-T. Kong, A. O. Govorov

Department of Physics and Astronomy, Ohio University, Athens, Ohio 45701, United States

E-mail: govorov@ohiou.edu

X.-T. Kong, Z. M. Wang

Institute of Fundamental and Frontier Sciences, University of Electronic Science and

Technology of China, Chengdu, 610054, China

E. Carbó-Argibay

International Iberian Nanotechnology Laboratory, Av. Mestre José Veiga s/n, 4715-330 Braga, Portugal

M. Comesaña-Hermo

Université de Paris, ITODYS, CNRS, UMR 7086, 15 rue J-A de Baïf, F-75013 Paris, France

E-mail: miguel.comesana-hermo@u-paris.fr

Keywords: plasmonic photocatalysis, hot electron injection, plasmon energy transfer, hybrid nanomaterials, photocatalytic hydrogen generation

Important efforts are currently under way in order to implement plasmonic phenomena in the growing field of photocatalysis, striving for improved efficiency and reaction selectivity. A significant fraction of such efforts has been focused on distinguishing, understanding and enhancing specific energy transfer mechanisms from plasmonic nanostructures to their environment. Herein we report a synthetic strategy that brings together two of the main physical mechanisms driving plasmonic photocatalysis into an engineered system by rationally combining the photochemical features of energetic charge carriers and the electromagnetic field enhancement inherent to the plasmonic excitation. We do so by creating hybrid photocatalysts that integrate multiple plasmonic resonators in a single entity, controlling their joint 
contribution through spectral separation and differential surface functionalization. This strategy allows us to study the combination of different photosensitization mechanisms when activated simultaneously. Our results show that hot electron injection can be combined with an energy transfer process mediated by near-field interaction, leading to a significant increase of the final photocatalytic response of the material. In this manner, we overcome the limitations that hinder photocatalysis driven only by a single energy transfer mechanism, and move the field of plasmonic photocatalysis closer to energy-efficient applications. Furthermore, our multimodal hybrids offer a test system to probe the properties of the two targeted mechanisms in energyrelated applications such as the photocatalytic generation of hydrogen and open the door to wavelength-selective photocatalysis and novel tandem reactions.

\section{Introduction}

In the last decades, plasmonic metal nanoparticles (NPs) have gained relevance as photosensitizers given their ability to extend the photocatalytic efficiency of large bandgap semiconductors, such as $\mathrm{TiO}_{2}$, to a broader range of the electromagnetic spectrum. As a result, metal-semiconductor nanoarchitectures have been promoted to the forefront of the rapidly developing fields of photovoltaics, photochemistry and photoelectrochemistry. ${ }^{[1-6]}$ Along these lines, these hybrid nanosystems can be exploited in order to increase the overall performance of solar cells, ${ }^{[7-9]}$ drive different organic transformations, ${ }^{[10,11]}$ or boost water splitting efficiency. ${ }^{[12,13]}$

Plasmonic photosensitization of semiconductors results from the ability of metal NPs to support collective electronic oscillations, also known as localized surface plasmon resonances (LSPRs), excited upon light irradiation. The plasmonic excitations of coinage metals $(\mathrm{Ag}, \mathrm{Au}, \mathrm{Cu})$ are strong, narrow, and highly tunable through changes in the geometry of the NPs, leading to absorption signatures with high versatility that span through the visible and near-infrared (NIR) regions of the electromagnetic spectrum. ${ }^{[14]}$ Moreover, LSPRs confer these metallic objects with very high absorption cross-sections ${ }^{[15]}$ and the ability to behave as light concentrators by 
holding strong electromagnetic field enhancements at their surfaces, features that render them ideal candidates in sensing and theranostic applications. ${ }^{[16]}$ Once excited, plasmonic resonances can transfer their energy to a nearby acceptor thanks to the ability of metal NPs to create a population of excited "hot charge carriers" upon visible and/or NIR irradiation. ${ }^{[17-21]}$ The term "hot charge carriers" describes the non-thermal distribution of electrons and holes created upon optical excitation of a plasmonic resonator. Even though they are short-lived in nature $(<100$ fs), under continuous wave (CW) illumination they are being constantly excited, hence ensuring the formation of a steady-state population. Interestingly, hot carriers can be injected through the Schottky barrier created at a metal-semiconductor interface, avoiding relaxation through thermalization processes. ${ }^{[22,23]}$ This effect, known as hot electron injection (HEI), allows the electrons to propagate to the conduction band of the semiconductor, leading to a privileged catalytic site for reduction reactions, while the holes created below the Fermi level of the metal can be used to drive oxidation reactions. ${ }^{[24]}$

It is generally accepted that a correct band alignment and the formation of a physical interface between the metal and the semiconductor are essential parameters in order to obtain an efficient HEI process. ${ }^{[25]}$ However, the use of hot electrons as a powerful means to photosensitize large bandgap semiconductors faces important limitations. Firstly, plasmonic excitations are mostly composed of low energy electronic states, hence leading to a majority of charges not having sufficient energy to overcome the Schottky barrier. ${ }^{[26]}$ Accordingly, the number of hot electrons that can be extracted from a given plasmonic resonator is intrinsically limited. Moreover, even in those scenarios in which an efficient HEI across the Schottky barrier can be attained, another limitation related to the metal concentration is observed. Along these lines, when exceeding a certain metal concentration threshold, a further rise in the amount of photosensitizer induces a decrease in the photocatalytic efficiency. Previous reports explain this effect as a consequence of the electron-hole pair recombination under specific metal concentrations. ${ }^{[27-29]}$ In this case, 
metal components behave as recombination centers, providing an additional decay channel for the excited carriers in the semiconductor, reducing substantially the lifetime of the electronhole pairs that contribute to the overall photocatalytic activity.

Plasmonic photosensitization can be also achieved through mechanisms not involving a charge transfer between the different components. In fact, the electromagnetic field enhancement associated to a given plasmonic excitation can drive an enhanced photosensitization of a nearby acceptor such as a large bandgap semiconductor. ${ }^{[25]}$ The nature of this interaction, which will be referred to as plasmon-induced energy transfer (PET), is generally associated with the ability of noble metal NPs to focalize the incoming photons onto their immediate surfaces, hence increasing the local photonic density of states and as a result, the effective absorption crosssection of the acceptor. ${ }^{[30]}$ Other works discuss this interaction in terms of a dipole-dipole coupling established between the absorption signature of the plasmonic resonator and the excitation band of the semiconductor, leading to the non-radiative excitation of the latter. ${ }^{[31,32]}$ In contrast to HEI, PET processes can be active in geometries in which the plasmonic sensitizer and the semiconductor are separated by an insulating material. The optimum thickness of such insulator remains a source of discussion: while some works claim that the near-field effect becomes insignificant beyond $3 \mathrm{~nm},{ }^{[30]}$ others alternatively point out to an optimum distance of $10 \mathrm{~nm}$ by asserting that shorter distances may increase back-energy transfer from the semiconductor to the metal NP. ${ }^{[25]}$ Attending to the physical description of the PET effect, this optimal thickness should depend on different characteristics of the system. Among those, the strength and spatial extension of the near-field enhancement of the resonator are likely to be relevant factors given their sensitivity to the geometrical features of the NP, such as the surface curvature.

Different examples in the literature show that the photocatalytic activity of plasmonic NPs can be driven by thermal effects, hot charges or the electromagnetic field enhancement; depending 
on the chemical reaction under study, the chemical composition and morphology of the resonator and the illumination conditions. ${ }^{[33-35]}$ Moreover, the plasmonic photosensitization of a large bandgap semiconductor depends strongly on the surface functionalization of the metal and the relative disposition of the different materials forming the hybrid. ${ }^{[28,36,37]}$ Along these lines, the rational design of a composite material in which the relative importance of HEI and PET mechanisms could be carefully monitored, would represent a relevant accomplishment. ${ }^{[38]}$ Pre-designed cooperative effects between different mechanisms can allow for a more efficient photosensitization in systems currently suffering from the limitations inherent to each mechanism when operating independently. Among the few reports tackling this issue, Cushing and coworkers have developed a hybrid architecture in which HEI and PET mechanisms coexist. ${ }^{[39]}$ In this work, when both photosensitization mechanisms are allowed by the geometry of the system and the nature of the materials involved, the latter is found to be responsible for the majority of the photocatalytic activation of the semiconductor. The authors explain this result in terms of transfer efficiency: the near field effect efficiency being very similar to that of the direct excitation of the semiconductor while that of charge transfer is usually limited (vide supra). In a different work, You and coworkers reach similar conclusions when developing a theoretical model to characterize the relative importance of HEI and PET. ${ }^{[40]}$ The authors find that HEI exhibits a low efficiency as a result of the energy distribution of the hot electrons on the plasmonic metal. Even though these examples can work as a generic compass allowing the prediction of the relative importance of both processes in some systems, important issues concerning the cooperative integration of different photosensitization mechanisms and their combination towards the development of a hybrid photocatalyst with improved performances have yet to be addressed.

In this work we provide new insights into HEI and PET mechanisms when participating simultaneously in the photosensitization of a large bandgap semiconductor. With this aim, we present a new class of metal-semiconductor hybrids in which two different types of plasmonic 
resonators coexist. In this way the unique cooperative interactions found in these superstructures (Scheme 1) can be rationally predicted and engineered. Along these lines, the photocatalytic activity of a system in which HEI has reached its maximum photosensitization capacity can be enhanced by the controlled integration of a PET-operated pathway, moving this technology towards energy-efficient applications. As an example of their relevance, these distinctive photoactivation features have been implemented to maximize the solar-driven generation of hydrogen, a renewable-produced energy vector of particular interest in the current global energy context. ${ }^{[41]}$ Moreover, the combination of plasmonic resonators with welldifferentiated signatures can lead to multimodal hybrids with wavelength-selective photosensitization capabilities, opening the door to more advanced features such as photocatalytic tandem reactions. 


\section{a}

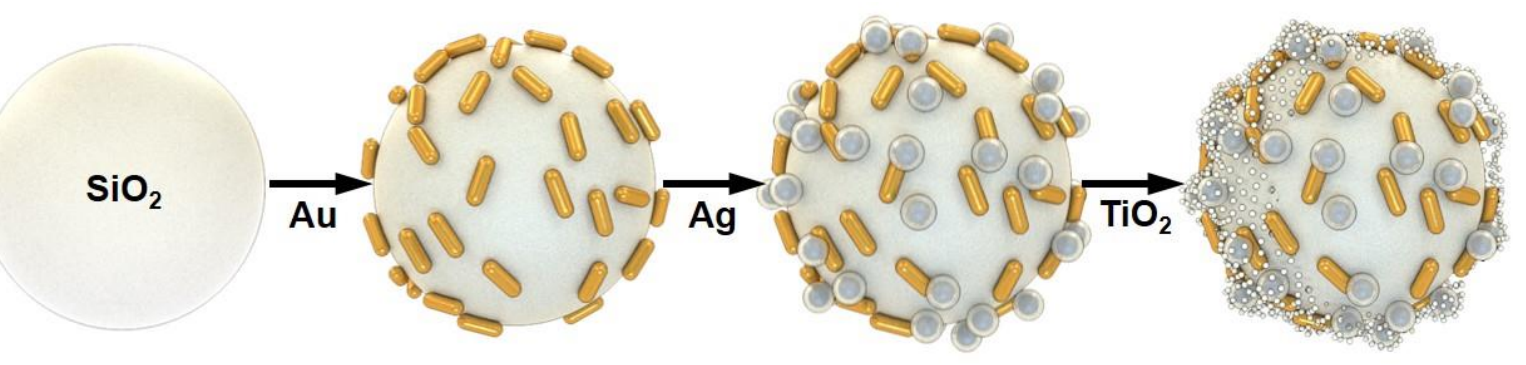

b
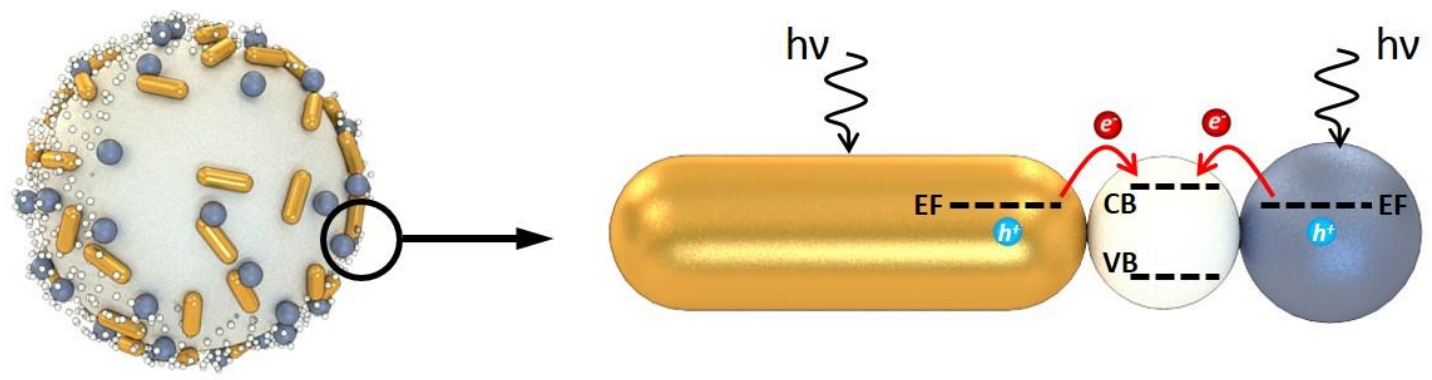

C
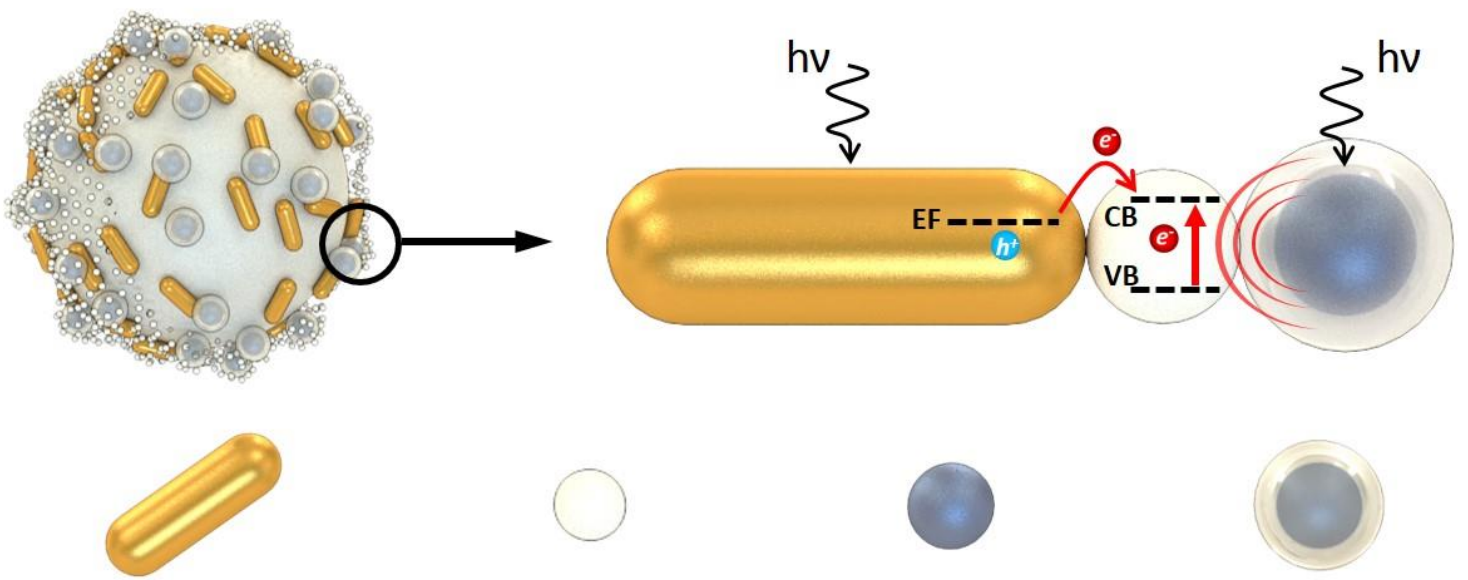

Au NR
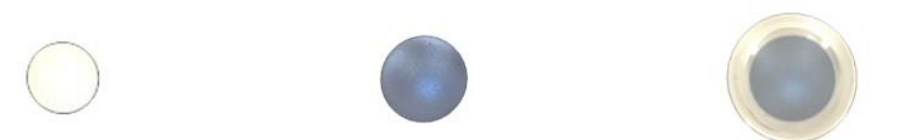

$\mathrm{TiO}_{2} \mathrm{NP}$

Ag NP

$\mathrm{Ag@SiO}{ }_{2} \mathrm{NP}$

Scheme 1. (a) Representation of the different steps of the assembly process used for the formation of the hybrid photocatalysts containing two different plasmonic resonators and $\mathrm{TiO}_{2}$ NPs. (b) Hybrid system composed by the combination of Au NRs and Ag NPs, being HEI the main photosensitization mechanism for both materials. (c) Hybrid system composed by the combination of $\mathrm{Au}$ NRs and $\mathrm{Ag}$ NPs coated with a $\mathrm{SiO}_{2}$ shell, being HEI and PET the key operating mechanisms, respectively.

\section{Results and discussion}

As previously mentioned, the physicochemical properties of a plasmonic object are particularly important to understand its interaction with a given semiconductor. Along these lines, the 
composition, morphology, size and surface functionalization of the metal will dictate the dominant photosensitization mechanism of the hybrid obtained and its overall efficiency. ${ }^{[28,37]}$ For this reason, three types of plasmonic NPs have been synthesized in order to fabricate the hybrid metal-semiconductor nanostructures reported herein. In this way, the different interactions between the metal and the semiconductor and thus, the distinctive photosensitization mechanisms established in these architectures, may be assessed separately (Figure S1). Accordingly, two plasmonic resonators showing a dominant HEI-based photosensitization have been selected: (i) Au nanorods (NRs) with an aspect ratio of 4 (53.8 nm $\mathrm{x} 13.3 \mathrm{~nm}$ ) and a longitudinal plasmon band centered at $818 \mathrm{~nm}$ in water and (ii) spherical $\mathrm{Ag}$ NPs with a $42 \mathrm{~nm}$ diameter displaying a plasmonic signature centered at $417 \mathrm{~nm}$ in water. For

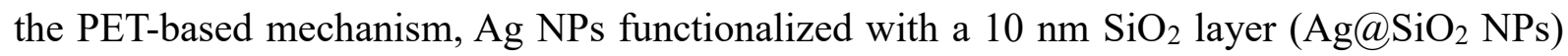
and showing a plasmon signature red-shifted to $436 \mathrm{~nm}$ have been selected.

In order to develop a model platform in which the interaction between the different components can be easily controlled and analyzed, we have made use of the layer-by-layer assembly protocol. ${ }^{[42]} \mathrm{SiO}_{2}$ particles with a $500 \mathrm{~nm}$ diameter have been used as colloidal substrates, providing a homogeneous interface for the assembly of the plasmonic objects and the semiconductor $\left(\mathrm{TiO}_{2} \mathrm{NPs}\right)($ Scheme 1$) .{ }^{[28]}$ More precisely, a first layer of the chosen plasmonic entity is deposited through electrostatic interactions, while a second layer of $5 \mathrm{~nm} \mathrm{TiO} 2$ (anatase phase) NPs is adsorbed in a subsequent step (see Experimental Section for details). Importantly, the hybrids formed in this way present high colloidal stability and are easily recoverable from the reaction medium by simple centrifugation. 

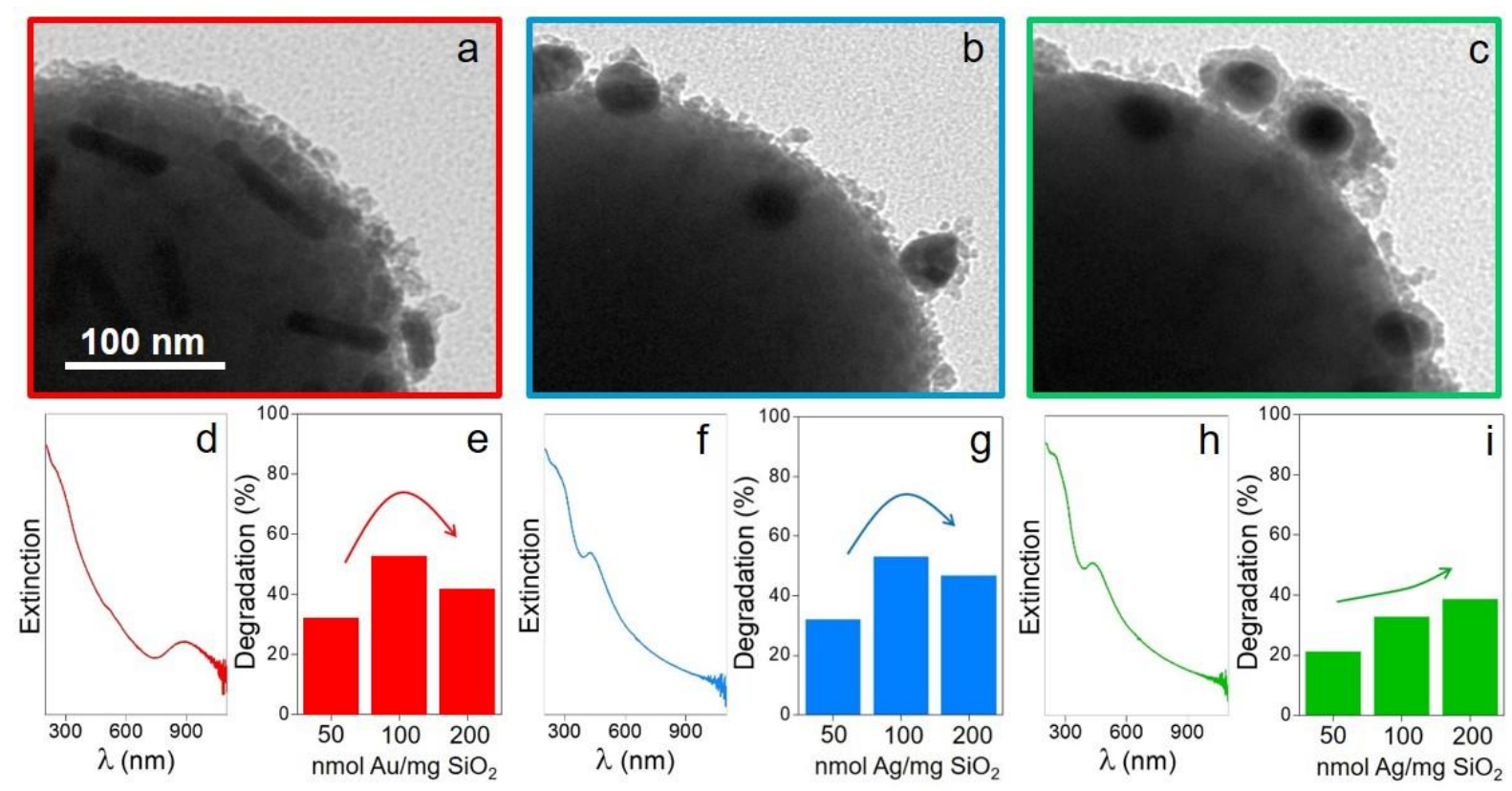

Figure 1. TEM images (a-c) and extinction spectra $(d, f$ and $h)$ of the nanohybrids formed with Au NRs (red), Ag NPs (blue) and Ag@ $\mathrm{SiO}_{2}$ NPs (green) with 100 nmol of metal/mg $\mathrm{SiO}_{2}$. (e, g and i) Photocatalytic activities with different metal concentrations using the degradation of $\mathrm{RhB}$ after an irradiation time of $180 \mathrm{~min}$ at $25^{\circ} \mathrm{C}$ with a solar simulator as model photochemical reaction.

Figure 1a-c and Figure S2 show TEM images of the hybrids obtained from the three different plasmonic entities. In all cases we can observe the formation of a smooth layer of $\mathrm{TiO}_{2} \mathrm{NPs}$ covering the entire surface of the composite, thus ensuring a good contact between the photosensitizer and the semiconductor. Interestingly, the extinction spectra of each nanohybrid shows an important scattering contribution coming from the colloidal $\mathrm{SiO}_{2}$ particles that hides the excitation band of $\mathrm{TiO}_{2}$. Nevertheless, the plasmonic signature of each metal NP is easily discernible (Figure 1d, f, h). The redshift of the main plasmonic signatures with respect to the original plasmon band of the metal NPs in aqueous solution can be ascribed to the modification of the refractive index when adsorbed onto the $\mathrm{SiO}_{2}$ spheres and coated with a thin layer of $\mathrm{TiO}_{2}$ NPs (Figure S3). In the particular case of the hybrids created with Au NRs, the longitudinal 
plasmon band is kept well below $\mathrm{h} \omega=1 \mathrm{eV}$, thus exciting carriers to energies that are sufficient for them to overcome the potential created between $\mathrm{Au}$ and $\mathrm{TiO}_{2}$.

In order to ascertain the photocatalytic features of the different hybrids, we have used the phototransformation of rhodamine $\mathrm{B}(\mathrm{RhB})$ in aqueous solutions as a model reaction. This dye is transformed into rhodamine 110 through the action of reactive oxygen species (ROS). ${ }^{[43]}$ Accordingly, the oxidation of water molecules by the holes generated through HEI and PET processes (Scheme 1) leads to the formation of hydroxyl radicals with high oxidative potential that are responsible for the photo-transformation of the dye. These radicals can also be generated at the conduction band of $\mathrm{TiO}_{2}$ by the multistep reduction of oxygen. ${ }^{[44]}$ The degradation of $\mathrm{RhB}$ can be quantified by following the decrease in absorbance $(\%$ of degradation) as a function of irradiation time. With this purpose, a solar simulator has been used as a light source, having an irradiation spectrum that covers the region between 350 and 2400 $\mathrm{nm}$. Moreover, the photocatalytic experiments are performed at $25{ }^{\circ} \mathrm{C}$, using a jacketed reactor in order to minimize any possible thermal contributions. Along these lines, disentanglement between thermal processes and carrier-induced catalysis is a major point to take into consideration in plasmon-induced photocatalysis. ${ }^{[19,45-47]}$ As a control experiment, the photocatalytic activity of $\mathrm{SiO}_{2}$ spheres functionalized solely with $\mathrm{TiO}_{2} \mathrm{NPs}$ has been tested (Figure S4). These objects present a reduced degradation of RhB after 180 min of irradiation $(12 \%)$ that comes from the direct activation of $\mathrm{TiO}_{2}$ with the UV irradiation of the solar simulator. The same photocatalytic reaction in the presence of $\mathrm{SiO}_{2}$ spheres functionalized only with Au NRs, Ag NPs or Ag@ $\mathrm{SiO}_{2}$ NPs (without semiconductor) lead to no degradation (Figure S4), evidencing that heat dissipation through plasmonic excitation alone cannot explain the photo-transformation of the probe. These results also demonstrate the absence of direct injection of carriers from the metal to the molecular species. Moreover, plasmonic objects in a HEI configuration could work as electron sinks, increasing charge separation lifetime in $\mathrm{TiO}_{2}$ 
and improving the photocatalytic activity. ${ }^{[48]}$ We excluded this competing contribution in complementary experiments by subtracting the direct activation of the semiconductor using filters that block light below $400 \mathrm{~nm}$ (vide infra).

We have verified the optimal amount of metal component needed for a maximum photosensitization of the semiconductor. To this end, the amount of photosensitizer has been adjusted while keeping constant the remaining parameters (i.e., same amount of $\mathrm{TiO}_{2}$ and $\mathrm{SiO}_{2}$ support). The photodegradation of $\mathrm{RhB}$ after $180 \mathrm{~min}$ of irradiation is presented in Figure 1e, $\mathrm{g}$ and $\mathrm{i}$ for hybrids containing $\mathrm{Au}$ NRs, $\mathrm{Ag} \mathrm{NPs}$ and $\mathrm{Ag} @ \mathrm{SiO}_{2} \mathrm{NPs}$, respectively (the photocatalytic profiles are presented in Figure S5). Along these lines, the two nanohybrids in which the plasmonic element forms a Schottky barrier with the semiconductor (Au NRs and naked Ag NPs) present an increase in the catalytic activity until a maximum level is reached (100 nmol of metal/mg SiO 2 ), leading in both cases to a degradation of 53\% (Figure 1e, g). Above this concentration, a partial loss in activity is observed. As previously stated, such effect can be ascribed to the role played by the metal NPs as recombination centers, acting as acceptors for excited charge carriers in the semiconductor, and thus leading to the faster annihilation of the electron-hole pair in a HEI process. Interestingly, only the hybrid in which HEI can be ruled out due to the insulating layer between the plasmonic NP and the semiconductor $\left(\mathrm{Ag} @ \mathrm{SiO}_{2}\right.$ NPs) presents an increase in the catalytic activity above that critical threshold, consistent with a PET process (Figure 1i).

The HEI and PET mechanisms can be modeled in terms of the hot electron generation rate (which serves as a proxy of the injection rates) and the electromagnetic field enhancement for each plasmonic resonator. As shown in previous works, the excitation of plasmonic bands creates the conditions for the excitation of large numbers of hot carriers in the metal NPs (Figure 2a). As mentioned above, these excited hot carriers differ from those participating in the collective plasmonic wave in that they have larger energies, up to the total energy of the 
incoming photons. This can occur due to the interaction of electrons and holes with the metal phonons ${ }^{[49]}$ and, importantly, the surface of the NPs. ${ }^{[50]}$ The rates of generation of such intraband hot carriers can be subdivided in two different components, according to whether they have enough energy to surpass the Schottky barrier separating the metal and semiconductor, with a height of $\sim 1 \mathrm{eV}$ in our case. ${ }^{[51,52]}$ The comparison of the hot carrier generation rates between Au NRs and Ag NPs in Figure 2a showcases again their spectral differences, and also identifies the Ag NPs as the better resonator for exciting the larger numbers of hot carriers. This contrast arises from the difference of material composition and the averaging of the strong longitudinal plasmonic mode of the Au NR with its significantly weaker transversal mode. ${ }^{[26]}$ On the other hand, $\mathrm{Au}$ NRs provide a stronger electromagnetic field enhancement around them, ${ }^{[28]}$ as quantified by averaging this magnitude in the volume immediately surrounding them (Figure $2 \mathrm{~b}$ ). This is caused by their strong and spatially extended main dipolar mode (see Figure S3d). Ag NPs also present a high density of hot charges and an electromagnetic field enhancement that coincide with the plasmonic signature of these objects (Figure 2a, b). In opposition to Au NRs, the latter effect appears in the vicinity of the absorption wavelengths of $\mathrm{TiO}_{2}$, implying that both effects (HEI and PET) can participate in the photoactivation mechanism. Although the average field enhancement outside of the Ag NP is significantly smaller than that of the Au NR due to the weaker dipolar moment of its plasmon, it is sufficient to achieve an effective enhancement above 1 order of magnitude. Even after considering an insulating $\mathrm{SiO}_{2}$ layer, the computed electromagnetic field of the $\mathrm{Ag} @ \mathrm{SiO}_{2} \mathrm{NPs}$ leads to an averaged enhancement factor of $\sim 6$ at the LSPR wavelength (Figure $2 b$ ). Although the light concentration at the surface of the $10 \mathrm{~nm} \mathrm{SiO} 2$ layer is reduced with respect to the naked $\mathrm{Ag} \mathrm{NP}$, it can still support the nonradiative excitation of the nearby semiconductor (Figure 2c). 

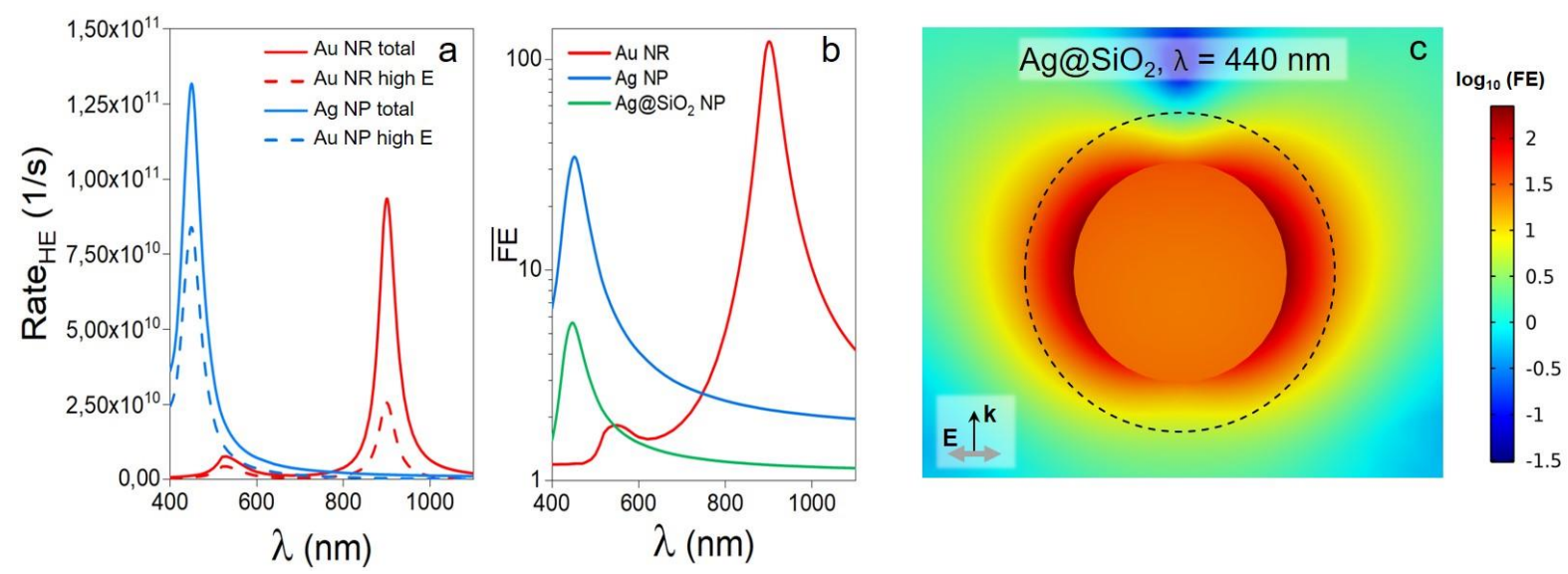

Figure 2. (a) Calculated rates of generation of hot electrons for both Au NRs and Ag NPs, in a homogenous dielectric medium simulating the environment with $\mathrm{SiO}_{2}$ and $\mathrm{TiO}_{2}$ in the hybrid. A second set of curves are included, indicating the rates of generation of high-energy hot electrons, or those with excess energies larger than the height of the Schottky barrier, taken in this calculation as $E_{\text {barrier }}=1 \mathrm{eV}$. (b) Spectra, in logarithmic scale, of the total field enhancement $\left(F E=|\mathbf{E}|^{2} / E_{0}^{2}\right)$ around the plasmonic systems, calculated as an average value within a layer of $10 \mathrm{~nm}$ surrounding the nanostructures. (c) Color map of the FE (in logarithmic scale) for the $\mathrm{Ag} @ \mathrm{SiO}_{2} \mathrm{NP}$. The black dashed line denotes the outer surface of the $10 \mathrm{~nm} \mathrm{SiO}_{2}$ coating, outside of which we can still observe regions where the enhancement surpasses 1 order of magnitude.

Once the optimum amount of plasmonic photosensitizer has been established $(100 \mathrm{nmol} / \mathrm{mg}$ $\mathrm{SiO}_{2}$ ), we proceeded with the synthesis of new hybrids with a higher degree of complexity. In this case, multiple plasmonic components are included in the same material, thus leading to a new scenario in which HEI and PET pathways can be combined rationally (Scheme 1). Moreover, the precise manipulation of the relative importance of both processes allows to create a model system in which their coexistence can be scrutinized. Along these lines, such a hybrid allows to understand the effect that the controlled integration of the PET mechanism will have in a system predominantly operated by a HEI-driven activation process that has already reached its maximum effective contribution (i.e., maximum metal concentration threshold). 
In order to synthesize such material, we have reproduced the assembly process previously described with the difference that two plasmonic catalysts $\left(100 \mathrm{nmol}\right.$ of metal $/ \mathrm{mg} \mathrm{SiO}_{2}$ of each one) are introduced in the same system. We have fabricated two complex hybrids with Au NRs and $\mathrm{Ag}$ NPs, the only difference between them being the surface functionalization of the latter (with or without an insulating $\mathrm{SiO}_{2}$ layer). Scanning transmission electron microscopy (STEM) and energy-dispersive X-ray spectroscopy (EDX) characterization demonstrates again the homogeneous distribution of all inorganic materials (plasmonic components and $\mathrm{TiO}_{2} \mathrm{NPs}$ ) onto the surface of the $\mathrm{SiO}_{2}$ beads (Figure 3a, b). Moreover, EDX mapping helps to identify the homogeneous $\mathrm{SiO}_{2}$ coating surrounding the $\mathrm{Ag}$ NPs in the second hybrid. The optical properties of both materials are very similar, with the main plasmonic contributions of Ag NPs and Au NRs clearly distinguishable. In this manner, the shoulder corresponding to Ag or $\mathrm{Ag} @ \mathrm{SiO}_{2} \mathrm{NPs}$ is located in the $420-430 \mathrm{~nm}$ range while the signature of Au NRs appears at $\sim 890 \mathrm{~nm}$ (Figure 3c, d). When the two plasmonic components present a "naked" surface state (Figure 3a) HEIbased activation from both materials is the major contribution responsible for the photocatalytic activity, while PET activation from Ag plays a secondary role. However, when the Ag component is functionalized with a $\mathrm{SiO}_{2}$ shell, it participates in the photoactivation by means of PET alone, making Au NRs the only HEI-operated component.

The catalytic activities of these complex hybrids have been analyzed once again using the photodegradation of RhB as a model reaction (Figure 3e). In this manner, we can compare their performances with respect to the reference hybrids containing a single plasmonic entity with $100 \mathrm{nmol}$ of metal/mg $\mathrm{SiO}_{2}$. The material composed by the combination of Au NRs and Ag NPs (without $\mathrm{SiO}_{2}$ shell) shows a reduction in its catalytic activity (44\% of degradation, grey column in Figure 3e) with respect to the systems in which Au NRs and Ag NPs are used independently ( $53 \%$ for each one). This difference can be explained as a result of the higher number of metal centers capable of capturing excited carriers in the $\mathrm{TiO}_{2}$ which contribute effectively to faster recombination kinetics in a photosensitization process. When $\mathrm{Ag} @ \mathrm{SiO}_{2}$ NPs are added in 
combination with Au NRs, an important increase in the catalytic activity is observed (88\% of degradation, black column in Figure 3e), matching the sum of both components when used separately (53\% with the Au NRs and 33\% for the $\mathrm{Ag} @ \mathrm{SiO}_{2} \mathrm{NPs}$ ). This system surpasses substantially the value reached through a purely HEI-driven mechanism $(53 \%$ for the system comprising $\mathrm{Au} \mathrm{NRs),} \mathrm{highlighting} \mathrm{the} \mathrm{absence} \mathrm{of} \mathrm{a} \mathrm{negative} \mathrm{effect} \mathrm{stemming} \mathrm{from} \mathrm{increasing}$ the metal concentration beyond the maximum activity threshold found when using Au NRs alone (i.e., above the threshold for faster recombination kinetics). More importantly, this finding proves that a purely PET-driven mechanism can expand the photosensitization of a metalsemiconductor hybrid in which HEI has been optimized and reached its maximum activity.

The comparative analysis between the different photocatalysts has also been carried on the photogeneration of hydrogen assisted by the decomposition of formic acid. Interestingly, this process produces the same trend that has been reported for the photodegradation of the organic dye (Figure 3f). On one hand, the combination of Au NRs and Ag NPs in the same hybrid leads to a clear decrease in the amount of photogenerated hydrogen, with a $\mathrm{H}_{2}$ production rate $(\mu \mathrm{mol}$ of $\mathrm{H}_{2}$ per mg of catalyst in 1 hour) of 0.21 vs. 0.32 and 0.3 when these plasmonic objects are used separately. On the other hand, the combination of Au NRs and $\mathrm{Ag} @ \mathrm{SiO}_{2} \mathrm{NPs}$ permits the cooperative activation of both HEI and PET processes, leading to a production rate of 0.43 that matches their activity when used independently (0.32 and 0.1 , respectively). Such findings corroborate the cooperative behavior of HEI and PET processes, regardless of the model photochemical reaction used, when the hybrid photocatalyst is rationally designed to combine both effects. 

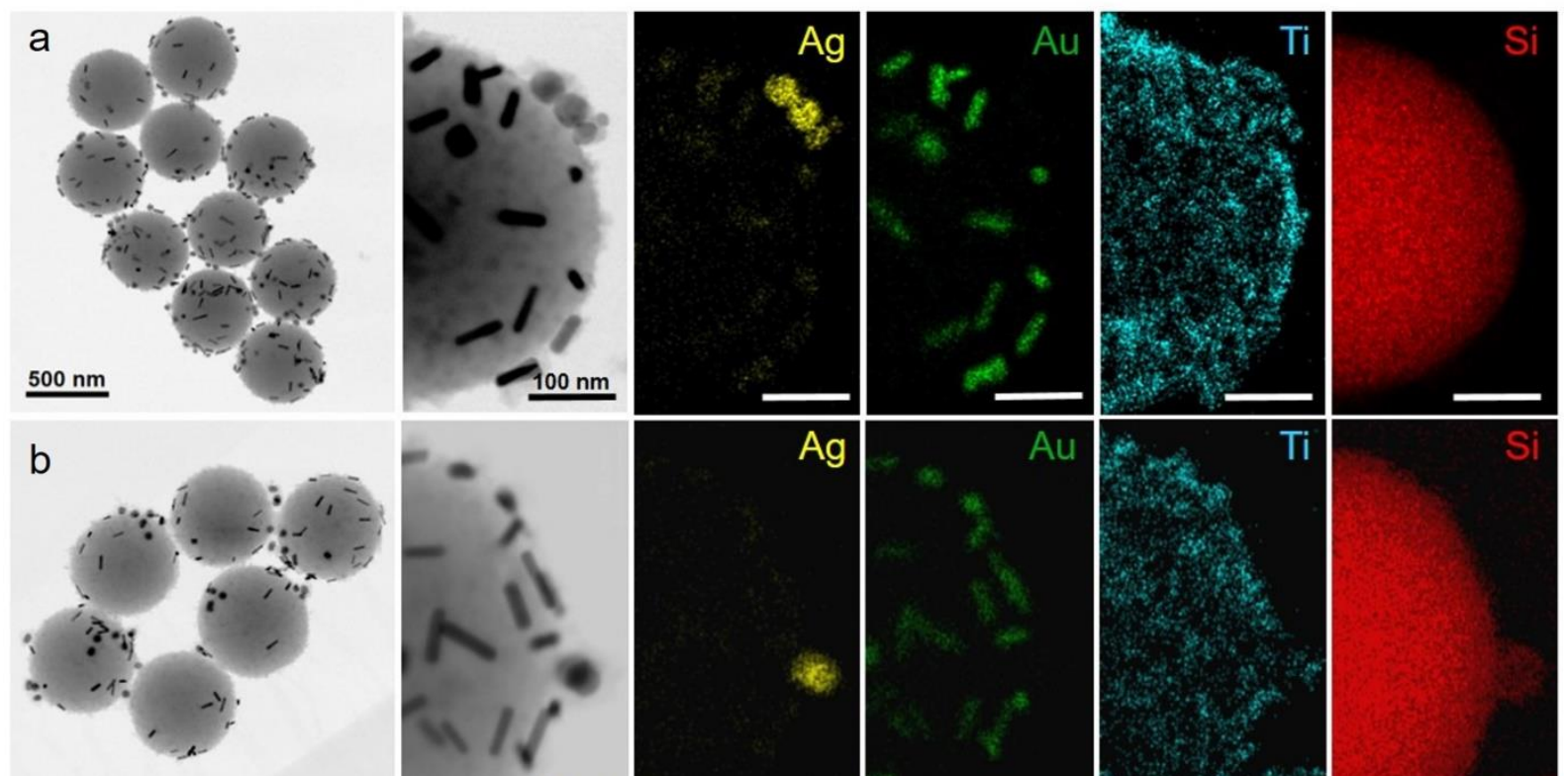

$500 \mathrm{~nm}$
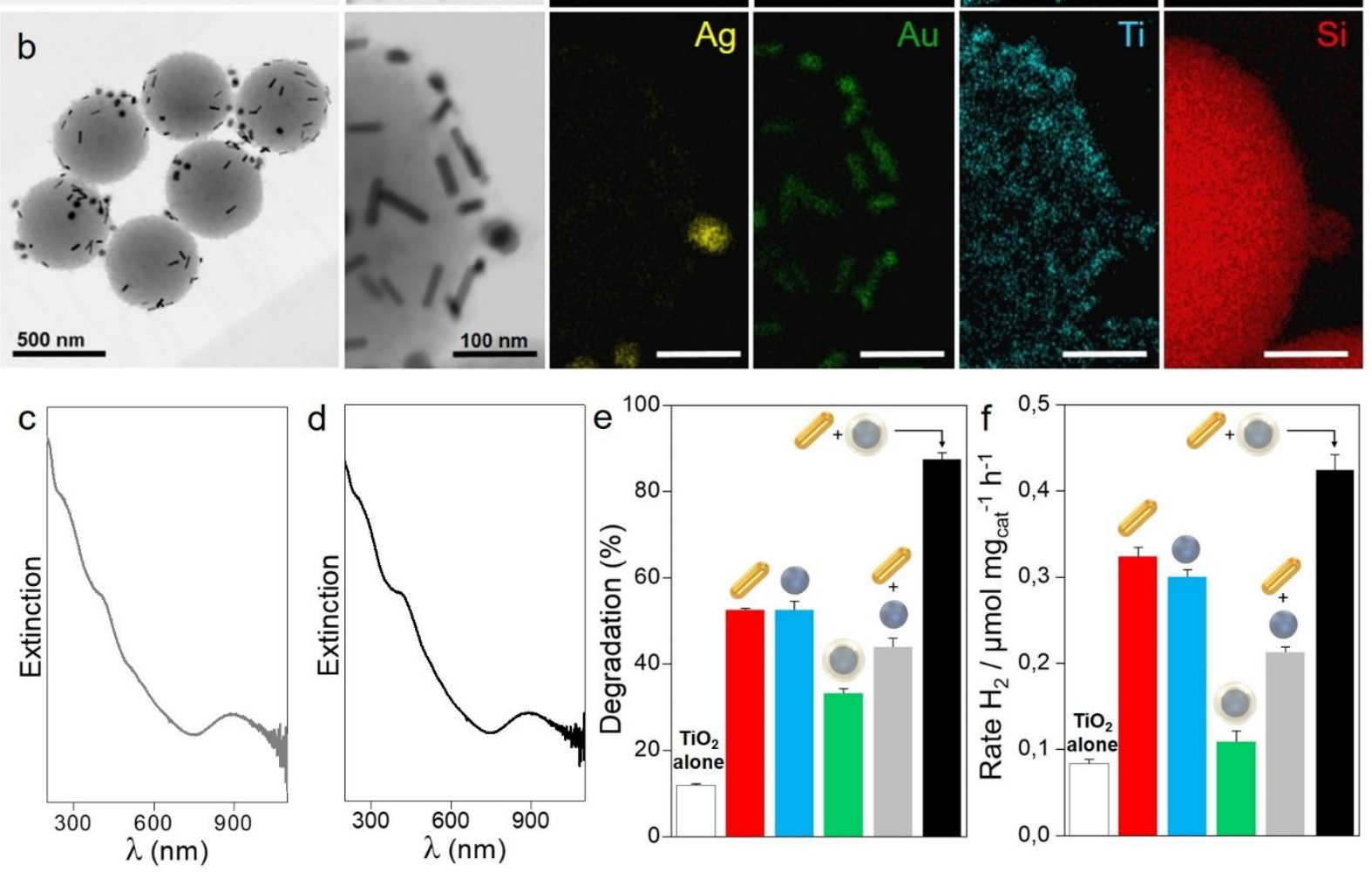

Figure 3. STEM images (bright field) and EDX mapping of the hybrid materials composed by (a) the assembly of Au NRs, $\mathrm{Ag}$ NPs and $\mathrm{TiO}_{2}$ NPs and (b) by the assembly of Au NRs, $\mathrm{Ag} @ \mathrm{SiO}_{2} \mathrm{NPs}$ and $\mathrm{TiO}_{2} \mathrm{NPs}$ onto the $\mathrm{SiO}_{2}$ beads. (c, d) Extinction spectra of the hybrid nanostructures based on $\mathrm{Au} \mathrm{NRs}+\mathrm{Ag} \mathrm{NPs}$ and $\mathrm{Au} \mathrm{NRs}+\mathrm{Ag} @ \mathrm{SiO}_{2}$ NPs, respectively. Comparison of the activity of the different hybrids synthesized in the present work in two different reactions: (e) photocatalytic degradation of $\mathrm{RhB}$ after $180 \mathrm{~min}$ of solar-simulated irradiation at $25^{\circ} \mathrm{C}$ and (f) photocatalytic $\mathrm{H}_{2}$ generation assisted by formic acid after $60 \mathrm{~min}$ of solar-simulated irradiation at $35{ }^{\circ} \mathrm{C}$. Plasmonic components: $100 \mathrm{nmol} / \mathrm{mg} \mathrm{SiO} 2$ of $\mathrm{Au} \mathrm{NRs}$ (red), $100 \mathrm{nmol} / \mathrm{mg} \mathrm{SiO}$ of Ag NPs (blue), 100 nmol/mg SiO 2 of $\mathrm{Ag} @ \mathrm{SiO}_{2}$ NPs (green), 100 $\mathrm{nmol} / \mathrm{mg} \mathrm{SiO} 2$ of $\mathrm{Au} \mathrm{NRs}+100 \mathrm{nmol} / \mathrm{mg} \mathrm{SiO} 2$ of Ag NPs (grey); $100 \mathrm{nmol} / \mathrm{mg} \mathrm{SiO}_{2}$ of $\mathrm{Au}$ 
$\mathrm{NRs}+100 \mathrm{nmol} / \mathrm{mg} \mathrm{SiO} 2$ of $\mathrm{Ag} @ \mathrm{SiO}_{2} \mathrm{NPs}$ (black). The white column in the left represents the reference sample with $\mathrm{SiO}_{2}$ particles solely functionalized with $\mathrm{TiO}_{2} \mathrm{NPs}$. The amount of $\mathrm{TiO}_{2}$ is kept constant in all the hybrids. All experiments have been performed at least three times (error bars are included).

We can go one step forward in the analysis of the catalytic activity of the complex hybrids containing two different plasmonic resonators by isolating the photoexcitation of each component, disentangling the performance of the different metals independently. In order to do so, two different glass-colored filters have been used to isolate different excitation ranges (400$700 \mathrm{~nm}$ targeting the resonance of the $\mathrm{Ag}$ components and 700-2400 $\mathrm{nm}$ targeting the longitudinal plasmon band of Au NRs (Figure 4a, c and S6). In the latter case, previous studies have shown that the transversal mode of Au NRs plays a negligible role on the photoactivation of $\mathrm{TiO}_{2},{ }^{[28]}$ leaving the longitudinal one in the NIR as the only strong contribution. In the case of the Ag NPs in the complex Ag NP-Au NR nanohybrid an important reduction in the photocatalytic activity is observed with respect to the activation of the individual resonator with the entire solar spectrum (39\% vs. 53\%). A similar effect is observed when the Au NRs are excited with the NIR component $(\lambda$ : $700-2400 \mathrm{~nm})$, leading to a total degradation of $34 \%$ (Figure 4b). When the same experiment is run again with the hybrid nanostructures containing Au NRs and Ag@ $\mathrm{SiO}_{2}$ NPs we observe a similar trend (Figure 4c). In this case, when the visible component is used (400-700 nm) we observe that the original $33 \%$ photodegradation observed for $\mathrm{Ag} @ \mathrm{SiO}_{2} \mathrm{NPs}$ alone with the entire solar spectrum has been reduced to 23\%. Similarly, the degree of degradation of $\mathrm{RhB}$ is reduced to $41 \%$ when only the NIR region (700-2400 nm) is used (Figure 4d). The partial decrease in photocatalytic activity with respect to the original values of the isolated plasmonic resonators can be explained by two major factors. Firstly, the photocatalytic activities obtained when using the optical filters are underestimated since their use induces a $\sim 10 \%$ decrease in light transmittance (Figure S6). Secondly, the use of the filters leads to the loss of the direct photoexcitation of $\mathrm{TiO}_{2}$ from the UV segment of the solar simulator 
( $12 \%$ degradation after $180 \mathrm{~min})$. Besides these experimental dissimilarities, the wavelengthdependent activation indicates that the hybrid photocatalysts allow for the selective activation of the plasmonic resonators, an operational feature with great potential when aiming at developing novel processes in wavelength-selective photochemistry or tandem photochemical reactions.
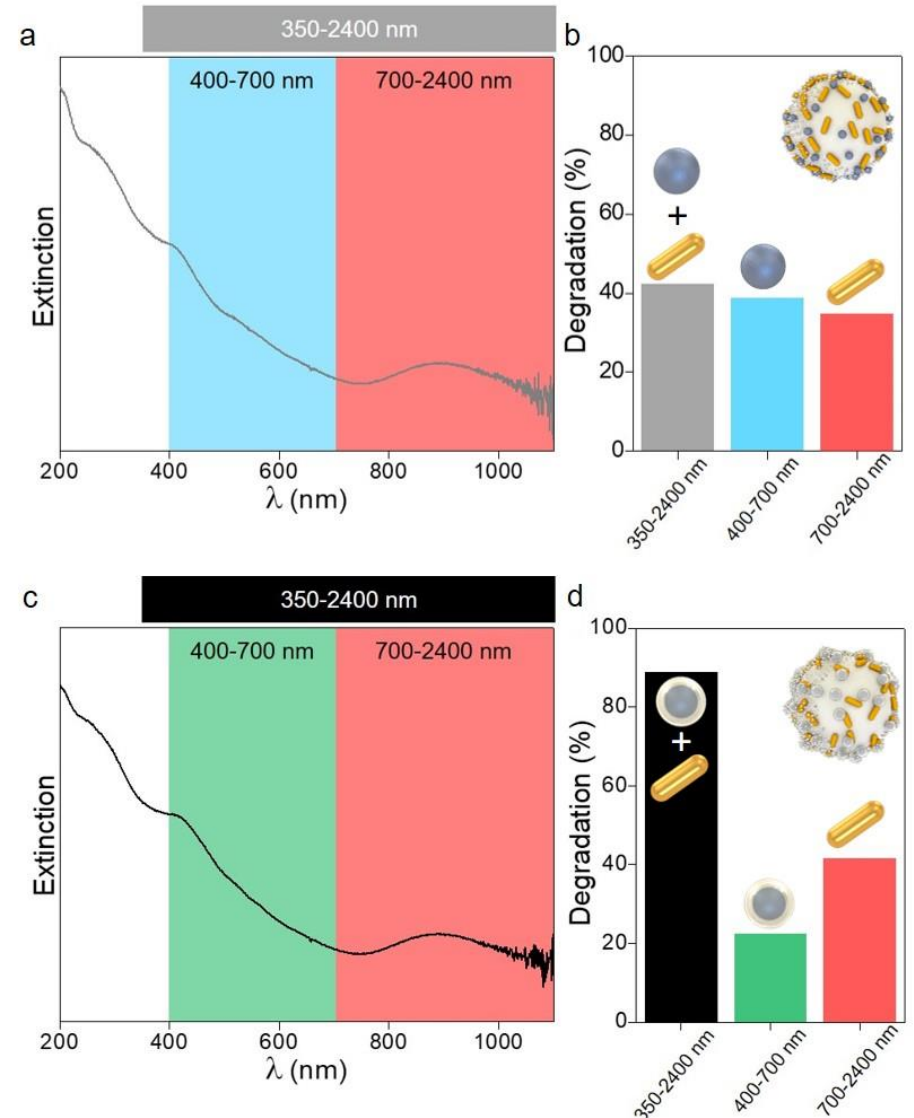

Figure 4. (a) Extinction spectra of the hybrid nanostructures containing Au NRs and Ag NPs and (b) their photocatalytic degradation capabilities of $\mathrm{RhB}$ with different excitation ranges. (c) Extinction spectra of the hybrids composed by the combination of Au NRs and $\mathrm{Ag} @ \mathrm{SiO}_{2} \mathrm{NPs}$ and (d) their photocatalytic degradation capabilities of RhB with different excitation ranges. In both cases the irradiation with a solar simulator proceeds at $25^{\circ} \mathrm{C}$ for $180 \mathrm{~min}$.

\section{Conclusions}

A layer-by-layer assembly protocol has been developed in order to produce complex photocatalytic hybrid nanostructures in which multiple plasmonic resonators are combined 
together with a large bandgap semiconductor. In this way, a distinctive scenario in which multiple photoactivation mechanisms are available in a single catalyst can be achieved. This approach allows the simultaneous operation of HEI and PET processes, producing an overall increase in the photocatalytic response of the system compared to that found when exploiting these mechanisms separately. The enhanced photosensitization of the semiconductor is the result of the simultaneous integration of both activation processes, overcoming current limitations in the use of hot charges in photochemical reactions. We believe that these results will contribute to a better understanding of the photocatalytic features of plasmonic metals, hence paving the way to the implementation of these materials as catalysts in a wide array of green and cost-effective chemical processes using sunlight as sole energy source.

\section{Experimental Section/Methods}

Materials: Tetrachloroauric acid $\left(\mathrm{HAuCl}_{4} \cdot 3 \mathrm{H}_{2} \mathrm{O}\right)$, hexadecyltrimethylammonium bromide (CTAB), sodium borohydride $\left(\mathrm{NaBH}_{4}\right)$, silver nitrate $\left(\mathrm{AgNO}_{3}\right)$, hydrochloric acid $(\mathrm{HCl}), \mathrm{L}-$ ascorbic acid (AA), poly(allylamine hydrochloride) (PAH, MW: $17500 \mathrm{~g} / \mathrm{mol}$ ), sodium chloride ( $\mathrm{NaCl}$ ), poly(styrenesulfonate) (PSS, MW: $14900 \mathrm{~g} / \mathrm{mol}$ ), tannic acid (TA), formic acid (FA), tetraethylorthosilicate 98\% (TEOS), ammonium hydroxide solution $28-30 \%$ $\left(\mathrm{NH}_{4} \mathrm{OH}\right)$ and sodium citrate $\left(\mathrm{Na}_{3} \mathrm{C}_{6} \mathrm{H}_{5} \mathrm{O}_{7}\right)$, were purchased from Sigma-Aldrich. $\mathrm{TiO}_{2}$ nanoparticles of $5 \mathrm{~nm}$ were purchased from Nanoamor. Pure grade ethanol and Milli-Q grade water were used in all preparations.

Synthesis and functionalization of $\mathrm{SiO}_{2}$ beads: Monodisperse $\mathrm{SiO}_{2}$ spheres (501 ( \pm 1$\left.) \mathrm{nm}\right)$ were prepared using a modified Stöber method. ${ }^{[53]}$ Typically, a TEOS solution $(1.7 \mathrm{~mL}, 1.2 \mathrm{M})$ was added to a solution containing ethanol $(18.12 \mathrm{~mL})$, ammonium hydroxide $(1.96 \mathrm{~mL})$, and water (3.21 mL). This mixture was stirred at room temperature for $2 \mathrm{~h}$. The excess of reagents was removed by three centrifugation-redispersion cycles with ethanol ( $2800 \mathrm{~g}, 20 \mathrm{~min})$. 
Subsequently, $\mathrm{PAH}$ was dissolved in a $0.5 \mathrm{M} \mathrm{NaCl}$ aqueous solution ( $\mathrm{pH} 5.0$ ) with a final polymer concentration of $1 \mathrm{mg} / \mathrm{mL}$. Then, $25 \mathrm{~mL}$ of the positively charged PAH solution were added to the $\mathrm{SiO}_{2} \mathrm{NPs}(20 \mathrm{mg})$ and stirred at room temperature for $30 \mathrm{~min}$. The excess of reagents was removed by three centrifugation-redispersion cycles with water (4000 g, $20 \mathrm{~min}$ ). Synthesis of Au nanorods (Au NRs) and PSS coating: Au NRs with localized surface plasmon resonance (LSPR) centered at $818 \mathrm{~nm}$ were synthesized by the seed-mediated growth method as described elsewhere. ${ }^{[54]}$ The dimensions obtained from TEM were $53.8( \pm 4.1) \mathrm{nm}$ of length and $13.3( \pm 1.1) \mathrm{nm}$ of thickness (aspect ratio 4.05). The Au NRs were subsequently coated with a layer of a negatively charged polyelectrolyte (PSS) in order to proceed to the deposition onto the positively charged PAH functionalized silica beads. ${ }^{[55]}$

Synthesis of $\mathrm{Ag}$ nanoparticles ( $\mathrm{Ag} \mathrm{NPS}$ ) and $\mathrm{SiO}_{2}$ coating: $\mathrm{Ag}$ NPs were synthesized as described elsewhere. ${ }^{[56]}$ The size was fixed at $42.7 \pm 3.4 \mathrm{~nm}$ by adjusting the concentration of $\mathrm{AgNO}_{3}, \mathrm{Na}_{3} \mathrm{C}_{6} \mathrm{H}_{5} \mathrm{O}_{7}$ and TA to $1 \mathrm{mM}, 2.45 \mathrm{mM}$ and $0.2 \mathrm{mM}$, respectively. The Ag NPs were subsequently coated with a thin layer of silica $(10.0 \pm 0.5 \mathrm{~nm})$ by following a previously published procedure. ${ }^{[57]}$

Au NRs@PSS,Ag NPs and Ag NPs@SiO 2 onto functionalized $\mathrm{SiO}_{2}$ beads: The solution of NPs (0.5, 1 and $2 \mathrm{~mL}$ of AuNRs@PSS $0.5 \mathrm{mM} ; 0.21,0.42$ and $0.84 \mathrm{~mL}$ of Ag NPs $1.19 \mathrm{mM} ; 0.5,1$ and $2 \mathrm{~mL}$ of Ag NPs@ $\left.\mathrm{SiO}_{2} 0.5 \mathrm{mM}\right)$ were added to $5 \mathrm{mg}$ of functionalized silica NPs $(50,100$ and 200 nmoles of metal per $\mathrm{mg}$ of $\mathrm{SiO}_{2}$ in all cases). The mixture was stirred at room temperature for $3 \mathrm{~h}$ and washed by three centrifugation-redispersion cycles (4000 g, $20 \mathrm{~min}$ ). The product was redispersed in $5 \mathrm{~mL}$ of water.

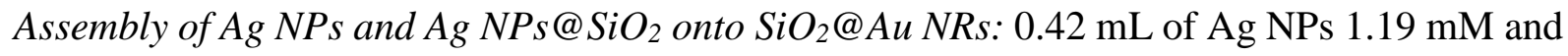
$1 \mathrm{~mL}$ of $\mathrm{Ag} \mathrm{NPs} @ \mathrm{SiO}_{2} 0.5 \mathrm{mM}$ were added to $5 \mathrm{mg}$ of $\mathrm{SiO}_{2} @ \mathrm{Au}$ NRs (100 nmoles of Au per $\mathrm{mg}$ of $\mathrm{SiO}_{2}$ ) functionalized with $\mathrm{PAH}$. The samples were stirred for $3 \mathrm{~h}$ and washed by three 
centrifugation-redispersion cycles with water (4000 g, $20 \mathrm{~min}$ ). Finally, each product was redispersed in $5 \mathrm{~mL}$ of water.

Deposition of $\mathrm{TiO}_{2}: 50 \mathrm{mg}$ of $\mathrm{TiO}_{2}(5 \mathrm{~nm})$ redispersed in $100 \mathrm{~mL}$ of a sodium citrate solution $(2,5 \mathrm{mM})$ was sonicated for $1 \mathrm{~h}$ with an ultrasonic tip. The aggregates of $\mathrm{TiO}_{2} \mathrm{NPs}$ were removed by centrifugation $(1340 \mathrm{~g}, 10 \mathrm{~min})$. Then, $5 \mathrm{~mL}$ of the $\mathrm{SiO}_{2} @ \mathrm{NPs}$ solution coated with another layer of $\mathrm{PAH}$ was added to $4 \mathrm{~mL}$ of the solution of $\mathrm{TiO}_{2}$ and stirred at room temperature for $60 \mathrm{~min}$. The excess of $\mathrm{TiO}_{2}$ was removed by three centrifugation-redispersion cycles (4000 $\mathrm{g}, 20 \mathrm{~min})$. Finally, the product was redispersed in $5 \mathrm{~mL}$ of water. The ratio $\mathrm{TiO}_{2} / \mathrm{SiO}_{2}$ was 2.1 $\pm 0.1 \mathrm{wt} \%$ (ICP analysis).

Chemical, structural and optical characterization: TEM images were obtained using a JEOL JEM 1010 transmission electron microscope operating at an acceleration voltage of $100 \mathrm{kV}$. STEM images (Bright Field detector) and EDX maps were obtained in a probe-corrected FEI ChemiSTEM electron microscope operating at $200 \mathrm{kV}$. UV-visible-NIR spectra were obtained with Hewlett-Packard HP8453 and Cary 5000 spectrophotometers.

Photocatalytic Study: The photocatalytic activity of the hybrids was evaluated with two different photochemical reactions: the degradation of rhodamine $\mathrm{B}(\mathrm{RhB})$ and the photogeneration of hydrogen assisted by the decomposition of FA.

Photodegradation of RhB: an aqueous solution of the dye is placed inside a controlled water bath at $25^{\circ} \mathrm{C}$ for 180 min under light irradiation from a LOT solar simulator (300 W Xe lamp) with $\lambda=350-2400 \mathrm{~nm}$. The solutions were prepared mixing $200 \mu \mathrm{L}$ of an aqueous solution of $\mathrm{RhB}(1 \mathrm{mM}), 4 \mathrm{~mL}$ of the hybrid solution $(1 \mathrm{mg} / \mathrm{mL})$ and $15.8 \mathrm{~mL}$ of Milli-Q water. The mixtures were stirred for $1 \mathrm{~h}$ in the dark to blend well and allow the adsorption-desorption equilibrium before the irradiation. Aliquots of $2.5 \mathrm{~mL}$ were taken with intervals of $30 \mathrm{~min}$ during the experiments in order to measure the variation in the absorbance. When necessary, a glass colored filter was introduced in the solar simulator (filter FM201 with $\lambda=400-700 \mathrm{~nm}$ 
and $90 \%$ of transmittance, to evaluate the photocatalytic activity in the visible region of the spectrum; filter $700 \mathrm{FH} 90$ with $\lambda=700-1100 \mathrm{~nm}$ and $90 \%$ of transmittance, to evaluate the photocatalytic activity in the NIR region of the spectrum) (Figure S6).

Photogeneration of hydrogen: Typically, $8 \mathrm{mg}$ of catalyst dispersed in $4.8 \mathrm{~mL}$ of water were mixed with $200 \mu \mathrm{L}$ of FA in a $13 \mathrm{~mL}$ reactor. The gases were purged with Ar for $2 \mathrm{~min}$ before sealing the flask. The dispersion was magnetically stirred inside a water bath at $35{ }^{\circ} \mathrm{C}$ under light irradiation with the solar simulator. After $1 \mathrm{~h}$, the gases were analyzed with an Agilent 7820A gas chromatographer in order to measure the volume of hydrogen generated.

Theoretical methods: The simulations obtained the optical response of the plasmonic systems within a classical electrodynamics framework, numerically solved using finite element methods (FEM) as implemented by the COMSOL commercial software package. The models of the NPs had sizes chosen in accord with the average ensemble sizes in the experiment (Figure S1) and with their optical profiles (Figure 1). The models for the nanorods are $53 \mathrm{~nm} \times 13 \mathrm{~nm}$ in size, while the Ag NPs are $45 \mathrm{~nm}$ in diameter, and their external $\mathrm{SiO}_{2}$ coating has a thickness of 10 nm. The complex permittivity of $\mathrm{Au}$ and $\mathrm{Ag}$ were extracted from published experimental datasets. ${ }^{[58,59]}$ The refractive index of the medium was taken as either that of water $\left(\mathrm{n}_{\mathrm{W}}=1.33\right)$, or a weighted average of the materials surrounding the plasmonic systems in the hybrids (resulting in $\mathrm{n}_{\text {hybrids }} \approx 1.5$ ). An incidence intensity of $3.6 \cdot 10^{3} \mathrm{~W} / \mathrm{cm}^{2}$ was assumed in the calculations. The field enhancement (FE) magnitude that is presented in the main text is defined as $E=|\mathbf{E}|^{2} / E_{0}^{2}$, where $E_{0}$ is the electric field's amplitude of the incoming radiation. The rates of generation of intraband hot carriers were calculated using a quantum formalism that accounts for the effect of the surface of the NPs, critical for allowing the excitation of high-energy hot carriers without violating the conservation of linear momentum. Full description of this formalism can be found in previous publications. ${ }^{[28,50,60]}$

\section{Supporting Information}

Supporting Information is available from the Wiley Online Library or from the author. 


\section{Acknowledgements}

M.C.-H. thanks CNRS for support. L.V.B. acknowledges support from the National Natural Science Foundation of China (Project No. 12050410252). A.O.G thanks the Nanoscale \& Quantum Phenomena Institute at Ohio University for its support. This work was funded by Ministerio de Ciencia e Innovación under grant PID2020-113704RB-I00, Xunta de Galicia/FEDER (IN607A 2018/5 and Centro Singular de Investigación de Galicia, Acc. 20192022, ED431G 2019-06), and European Union-ERDF (Interreg V-A - Spain-Portugal 0245_IBEROS_1_E, 0712_ACUINANO_1_E, and 0624_2IQBIONEURO_6_E, and Interreg Atlantic Area NANOCULTURE 1.102.531).

Received: ((will be filled in by the editorial staff))

Revised: ((will be filled in by the editorial staff)) Published online: ((will be filled in by the editorial staff))

\section{References}

[1] G. Tagliabue, J. S. DuChene, M. Abdellah, A. Habib, D. J. Gosztola, Y. Hattori, W.-H. Cheng, K. Zheng, S. E. Canton, R. Sundararaman, J. Sá, H. A. Atwater, Nat. Mater. 2020, 19, 1312.

[2] G. Tagliabue, A. S. Jermyn, R. Sundararaman, A. J. Welch, J. S. DuChene, R. Pala, A. R. Davoyan, P. Narang, H. A. Atwater, Nat. Commun. 2018, 9, 3394.

[3] S. Yu, A. J. Wilson, J. Heo, P. K. Jain, Nano Lett. 2018, 18, 2189.

[4] A. J. Wilson, P. K. Jain, Acc. Chem. Res. 2020, 53, 1773.

[5] U. Aslam, V. G. Rao, S. Chavez, S. Linic, Nat. Catal. 2018, 1, 656.

[6] S. Linic, S. Chavez, R. Elias, Nat. Mater. 2021, 20, 916.

[7] S. H. Lee, S. W. Lee, T. Oh, S. H. Petrosko, C. A. Mirkin, J. W. Jang, Nano Lett. 2018, $18,109$.

[8] Y. H. Jang, Y. J. Jang, S. Kim, L. N. Quan, K. Chung, D. H. Kim, Chem. Rev. 2016, 
$116,14982$.

[9] H. A. Atwater, A. Polman, Nat. Mater. 2010, 9, 205.

[10] S. Linic, P. Christopher, D. B. Ingram, Nat. Mater. 2011, 10, 911.

[11] X. Zhang, X. Li, D. Zhang, N. Q. Su, W. Yang, H. O. Everitt, J. Liu, Nat. Commun. 2017, 8,14542 .

[12] J. Liu, J. Feng, J. Gui, T. Chen, M. Xu, H. Wang, H. Dong, H. Chen, X. Li, L. Wang, Z. Chen, Z. Yang, J. Liu, W. Hao, Y. Yao, L. Gu, Y. Weng, Y. Huang, X. Duan, J. Zhang, Y. Li, Nano Energy 2018, 48, 44.

[13] S. Mubeen, J. Lee, N. Singh, S. Krämer, G. D. Stucky, M. Moskovits, Nat. Nanotechnol. 2013, 8, 247.

[14] L. M. Liz-Marzán, Langmuir 2006, 22, 32.

[15] P. K. Jain, K. S. Lee, I. H. El-Sayed, M. A. El-Sayed, J. Phys. Chem. B 2006, 110, 7238.

[16] J. Langer, D. Jimenez De Aberasturi, J. Aizpurua, R. A. Alvarez-Puebla, B. Auguié, J. J. Baumberg, G. C. Bazan, S. E. J. Bell, A. Boisen, A. G. Brolo, J. Choo, D. CiallaMay, V. Deckert, L. Fabris, K. Faulds, R. Goodacre, A. J. Haes, C. L. Haynes, C. Huck, M. Käll, J. Kneipp, N. A. Kotov, E. C. Le Ru, J.-F. Li, X. Y. Ling, M. Moskovits, K. Murakoshi, J.-M. Nam, Y. Ozaki, I. Pastoriza-Santos, J. Perez-Juste, J. Popp, A. Pucci, B. Ren, G. C. Schatz, T. Shegai, S. Schlücker, K. G. Thomas, Z.-Q. Tian, T. Vo-Dinh, K. A. Willets, C. Xu, Y. Xu, B. Zhao, L. M. Liz-Marzán, ACS Nano 2020, 14, 28.

[17] Y. Zhang, S. He, W. Guo, Y. Hu, J. Huang, J. R. Mulcahy, W. D. Wei, Chem. Rev. 2018, 118, 2927.

[18] G. V. Hartland, L. V. Besteiro, P. Johns, A. O. Govorov, ACS Energy Lett. 2017, 2, 1641.

[19] L. Zhou, D. F. Swearer, C. Zhang, H. Robatjazi, H. Zhao, L. Henderson, L. Dong, P. 
Christopher, E. A. Carter, P. Nordlander, N. J. Halas, Science 2018, 362, 69.

[20] D. B. Ingram, S. Linic, J. Am. Chem. Soc. 2011, 133, 5202.

[21] V. G. Rao, U. Aslam, S. Linic, J. Am. Chem. Soc. 2018, 141, 643.

[22] S. K. Cushing, C. J. Chen, C. L. Dong, X. T. Kong, A. O. Govorov, R. S. Liu, N. Wu, ACS Nano 2018, 12, 7117.

[23] J. R. M. Saavedra, A. Asenjo-Garcia, F. J. García de Abajo, ACS Photonics 2016, 3, 1637.

[24] E. Pensa, J. Gargiulo, A. Lauri, S. Schlücker, E. Cortés, S. A. Maier, Nano Lett. 2019, 19, 1867.

[25] T. Tatsuma, H. Nishi, T. Ishida, Chem. Sci. 2017, 8, 3325.

[26] L. Chang, L. V. Besteiro, J. Sun, E. Y. Santiago, S. K. Gray, Z. Wang, A. O. Govorov, ACS Energy Lett. 2019, 4, 2552.

[27] A. Bumajdad, M. Madkour, Phys. Chem. Chem. Phys. 2014, 16, 7146.

[28] A. Sousa-Castillo, M. Comesaña-Hermo, B. Rodríguez-González, M. Pérez-Lorenzo, Z. Wang, X.-T. Kong, A. O. Govorov, M. A. Correa-Duarte, J. Phys. Chem. C 2016, $120,11690$.

[29] S. Sakthivel, M. V. Shankar, M. Palanichamy, B. Arabindoo, D. W. Bahnemann, V. Murugesan, Water Res. 2004, 38, 3001.

[30] R. Asapu, N. Claes, R. G. Ciocarlan, M. Minjauw, C. Detavernier, P. Cool, S. Bals, S. W. Verbruggen, ACS Appl. Nano Mater. 2019, 2, 4067.

[31] J. Li, S. K. Cushing, F. Meng, T. R. Senty, A. D. Bristow, N. Wu, Nat Phot. 2015, 9, 601.

[32] S. K. Cushing, J. Li, F. Meng, T. R. Senty, S. Suri, M. Zhi, M. Li, A. D. Bristow, N. Wu, J. Am. Chem. Soc. 2012, 134, 15033.

[33] C. Zhan, X. J. Chen, Y. F. Huang, D. Y. Wu, Z. Q. Tian, Acc. Chem. Res. 2019, 52, 2784. 
[34] R. Kamarudheen, G. J. W. Aalbers, R. F. Hamans, L. P. J. Kamp, A. Baldi, ACS Energy Lett. 2020, 5, 2605.

[35] R. Kamarudheen, G. W. Castellanos, L. P. J. Kamp, H. J. H. Clercx, A. Baldi, ACS Nano 2018, 12, 8447.

[36] N. Wu, Nanoscale 2018, 10, 2679.

[37] Y. Negrín-Montecelo, M. Comesaña-Hermo, L. K. Khorashad, A. Sousa-Castillo, Z. Wang, M. Pérez-Lorenzo, T. Liedl, A. O. Govorov, M. A. Correa-Duarte, ACS Energy Lett. 2020, 5, 395.

[38] S. K. Cushing, N. Wu, J. Phys. Chem. Lett. 2016, 7, 666.

[39] S. K. Cushing, J. Li, J. Bright, B. T. Yost, P. Zheng, A. D. Bristow, N. Wu, J. Phys. Chem. C 2015, 119, 16239.

[40] X. You, S. Ramakrishna, T. Seideman, J. Chem. Phys. 2018, 149, 174304.

[41] Z. Wang, C. Li, K. Domen, Chem. Soc. Rev. 2019, 48, 2109.

[42] F. Caruso, R. A. Caruso, H. Möhwald, Science 1998, 282, 1111.

[43] A. Sousa-Castillo, J. R. J. R. J. Couceiro, M. Tomás-Gamasa, A. Mariño-López, F. López, W. Baaziz, O. Ersen, M. Comesaña-Hermo, J. L. J. L. Mascareñas, M. A. M. A. Correa-Duarte, M. Tomás Gamasa, A. Mariño-López, F. López, W. Baaziz, O. Ersen, M. Comesaña-Hermo, J. L. J. L. Mascareñas, M. A. M. A. Correa-Duarte, Nano Lett. 2020, 20, 7068 .

[44] M. Buchalska, M. Kobielusz, A. Matuszek, M. Pacia, S. Wojtyła, W. Macyk, ACS Catal. 2015, 5, 7424.

[45] G. Baffou, I. Bordacchini, A. Baldi, R. Quidant, Light Sci. Appl. 2020, 9, 108.

[46] L. Mascaretti, A. Naldoni, J. Appl. Phys. 2020, 128, 041101.

[47] S. Rej, L. Mascaretti, E. Y. Santiago, O. Tomanec, S. Kment, Z. Wang, R. Zboril, P. Fornasiero, A. O. Govorov, A. Naldoni, ACS Catal. 2020, 10, 5261.

[48] P. V. Kamat, J. Phys. Chem. C 2002, 106, 7729. 
[49] A. M. Brown, R. Sundararaman, P. Narang, W. A. Goddard, H. A. Atwater, ACS Nano 2016, $10,957$.

[50] E. Y. Santiago, L. V. Besteiro, X.-T. Kong, M. A. Correa-Duarte, Z. Wang, A. O. Govorov, ACS Photonics 2020, 7, 2807.

[51] N. Szydlo, R. Poirier, J. Appl. Phys. 1980, 51, 3310.

[52] P. Chinnamuthu, J. C. Dhar, A. Mondal, A. Bhattacharyya, N. K. Singh, J. Phys. D. Appl. Phys. 2012, 45, 135102.

[53] W. Stöber, A. Fink, E. Bohn, J. Colloid Interface Sci. 1968, 26, 62.

[54] L. Scarabelli, A. Sánchez-Iglesias, J. Pérez-Juste, L. M. Liz-Marzán, J. Phys. Chem. Lett. 2015, 6, 4270.

[55] I. Pastoriza-Santos, J. Pérez-Juste, L. M. Liz-Marzán, Chem. Mater. 2006, 18, 2465.

[56] N. G. Bastús, F. Merkoçi, J. Piella, V. Puntes, Chem. Mater. 2014, 26, 2836.

[57] C. Fernández-López, C. Mateo-Mateo, R. A. Álvarez-Puebla, J. Pérez-Juste, I. Pastoriza-Santos, L. M. Liz-Marzán, Langmuir 2009, 25, 13894.

[58] P. B. Johnson, R. W. Christy, Phys. Rev. B 1972, 6, 4370.

[59] E. D. Palik, Handbook of Optical Constants of Solids (Volume 2), Academic Press Inc., 1991.

[60] L. V. Besteiro, X.-T. Kong, Z. Wang, G. Hartland, A. O. Govorov, ACS Photonics 2017, 4, 2759. 
We report the synthesis of hybrid plasmonic photocatalysts in which the two most important physical mechanisms behind plasmon-induced photocatalysis (energetic charge carriers and electromagnetic field enhancement) can be activated simultaneously. Our results show that hot electron injection can be combined with an energy transfer process, leading to an important increase of the final photocatalytic response of the system.

Y. Negrín-Montecelo, X.-T. Kong, L. V. Besteiro, E. Carbó-Argibay, Z. M. Wang, M. PérezLorenzo, A. O. Govorov,* M. Comesaña-Hermo* and M. A. Correa-Duarte*

Multimodal Plasmonic Hybrids: Efficient and Selective Photocatalysts

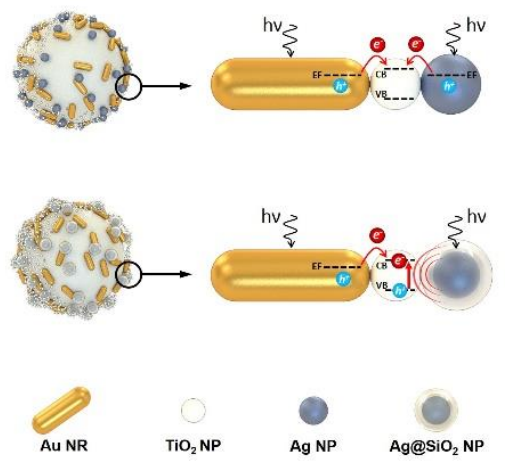




\section{Supporting Information}

\section{Multimodal Plasmonic Hybrids: Efficient and Selective Photocatalysts}

Yoel Negrín-Montecelo, Xiang-Tian Kong, Lucas V. Besteiro, Enrique Carbó-Argibay, Zhiming M. Wang, Moisés Pérez-Lorenzo, Alexander O. Govorov, * Miguel ComesañaHermo* and Miguel A. Correa-Duarte*
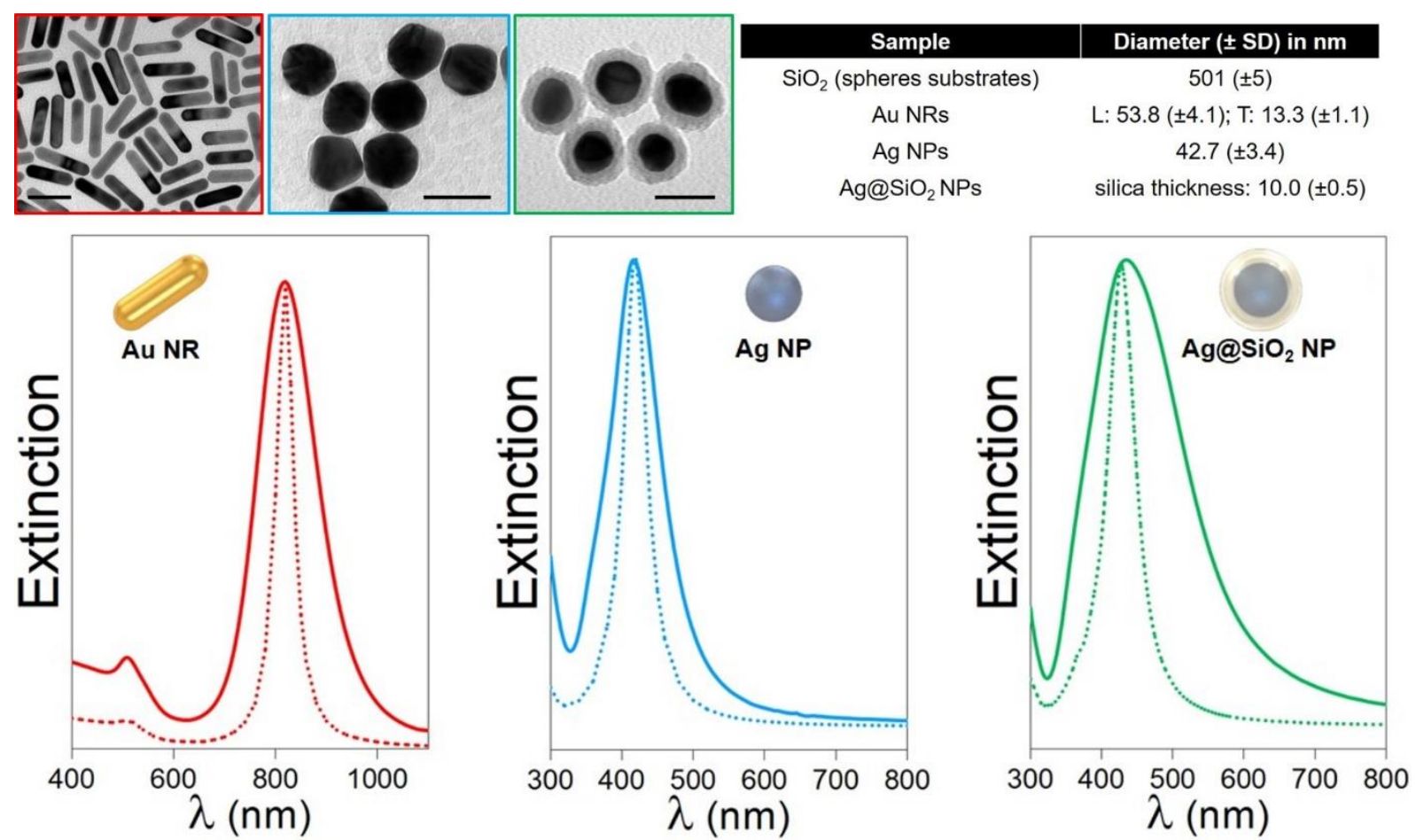

Figure S1. TEM images, dimensions and experimental (solid) and theoretical (dotted) extinction spectra of the individual components used for the formation of the hybrids: Au NRs (red), Ag NPs (blue) and Ag@ $\mathrm{SiO}_{2}$ NPs (green). Scale bars: $50 \mathrm{~nm}$. 

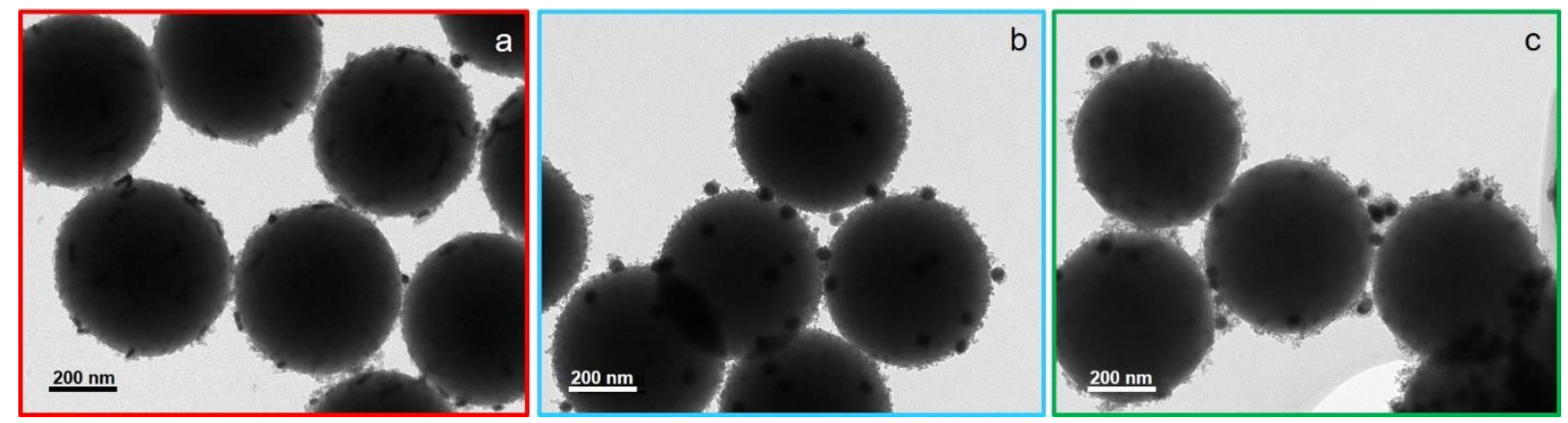

Figure S2. TEM (a-c) images of the hybrids formed by the adsorption of $\mathrm{TiO}_{2} \mathrm{NPs}_{\text {and }} \mathrm{Au}$ NRs (red), Ag NPs (blue) and $\mathrm{Ag} @ \mathrm{SiO}_{2} \mathrm{NPs}$ (green) onto $\mathrm{SiO}_{2}$ spheres.
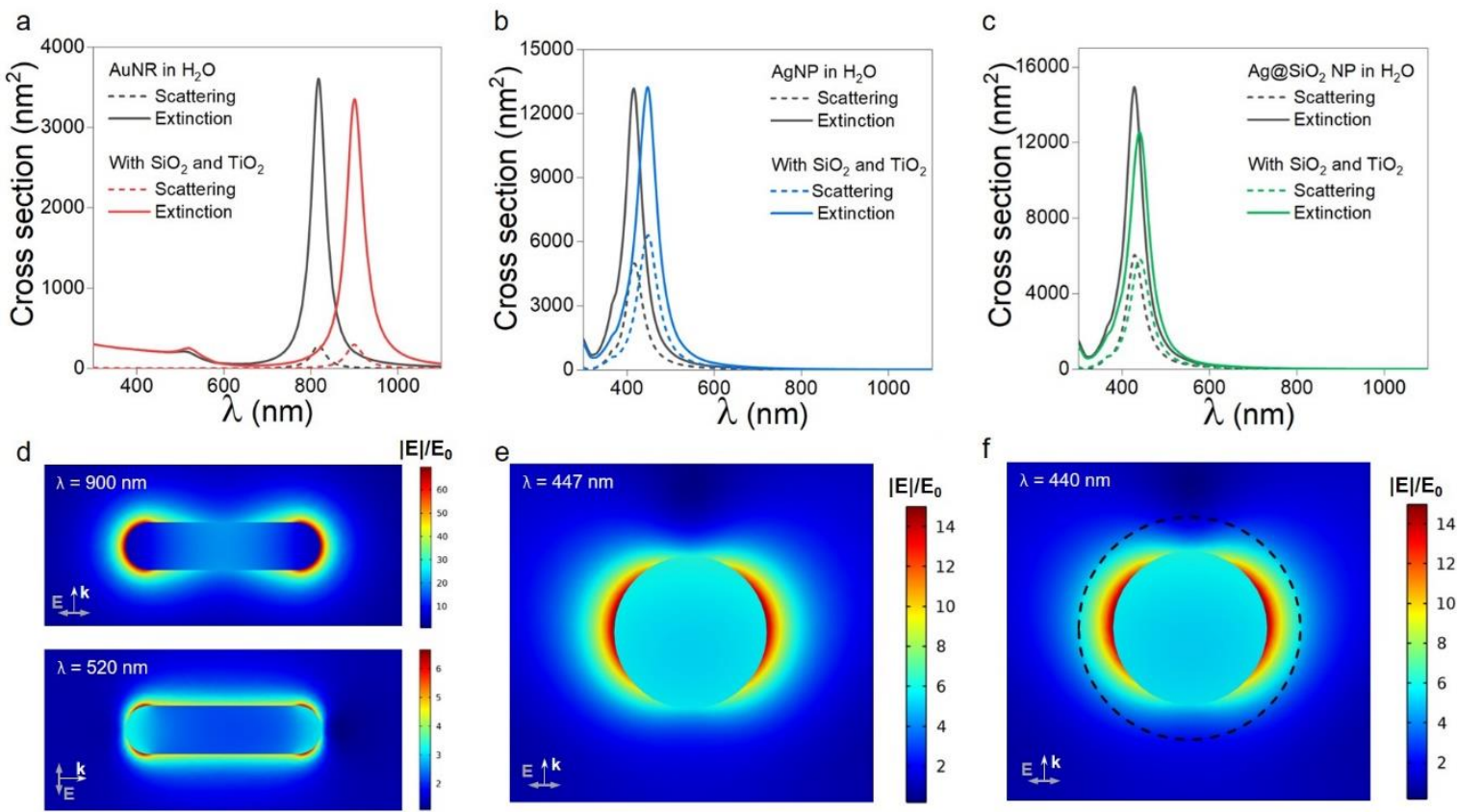

Figure S3. Theoretical results for the three type of plasmonic NPs considered in this work: $\mathrm{Au}$ NRs (a, d) Ag NPs (b, e), and $\mathrm{Ag} @ \mathrm{SiO}_{2}$ NPs (c, f). Panels a-c show extinction and scattering cross-sections in two situations: aqueous solution and in a homogeneous solution with permittivity chosen to mimic a mixture of $\mathrm{SiO}_{2}, \mathrm{TiO}_{2}$ and water. Panels d-f show the electric field distribution of the main plasmonic modes of these structures, taken at the resonant wavelength and in simulations reproducing the hybrid systems with $\mathrm{SiO}_{2}$ and $\mathrm{TiO}_{2}$. The dashed line in panel $\mathrm{f}$ marks the outer surface of the $\mathrm{SiO}_{2}$ layer. 


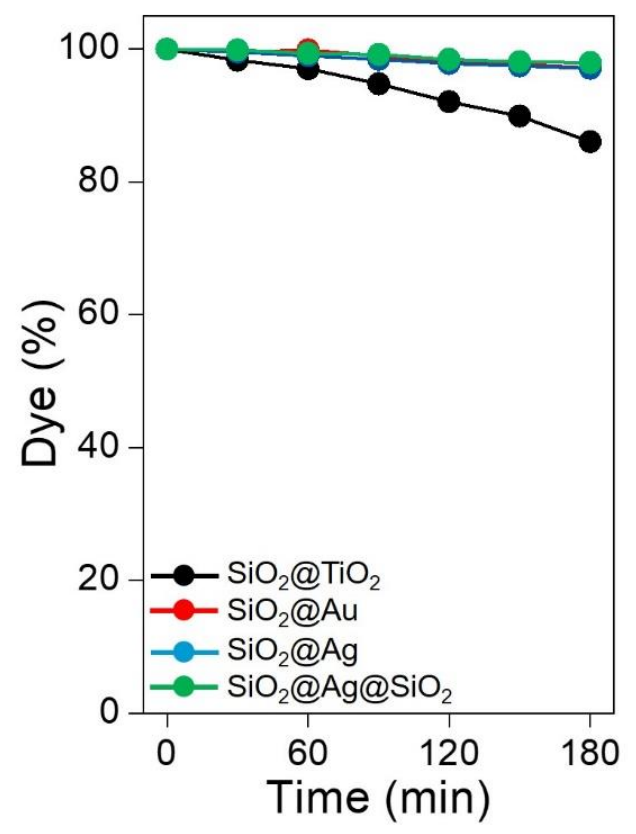

Figure S4. Photocatalytic degradation profiles of $\mathrm{RhB}$ in the presence of $\mathrm{SiO}_{2} \mathrm{NPs}$ functionalized either with $\mathrm{TiO}_{2}$ NPs (black), Au NRs (100 nmol/mg SiO 2 ; red), Ag NPs (100 nmol/mg SiO 2 ; blue) or Ag@ $\mathrm{SiO}_{2} \mathrm{NPs}$ (100 nmol/mg SiO 2 ; green). Excitation range: $350-2400$ nm.
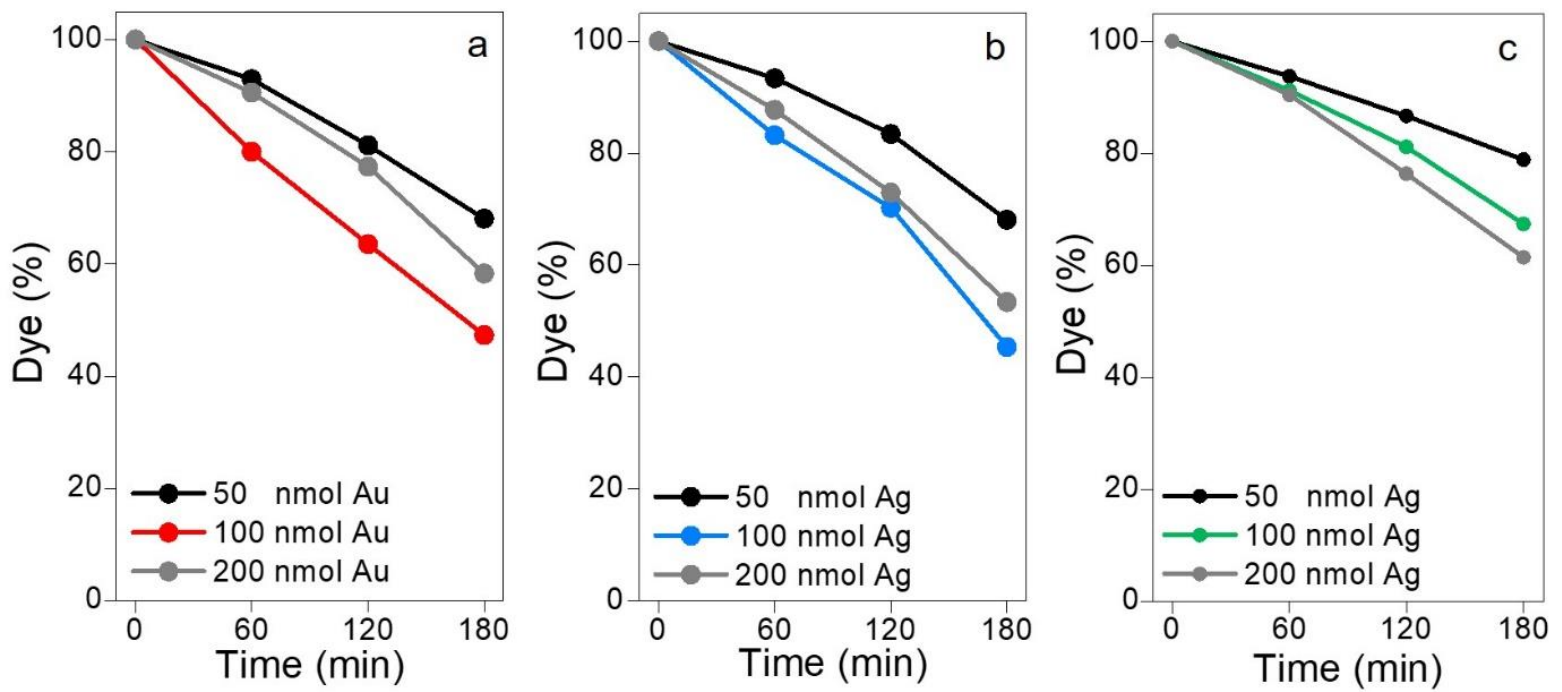

Figure S5. Photocatalytic profiles of the hybrids formed by the adsorption of $\mathrm{TiO}_{2} \mathrm{NPs}$ and different amounts of Au NRs (a), Ag NPs (b) and $\mathrm{Ag} @ \mathrm{SiO}_{2} \mathrm{NPs}$ (c) onto $\mathrm{SiO}_{2}$ spheres. 


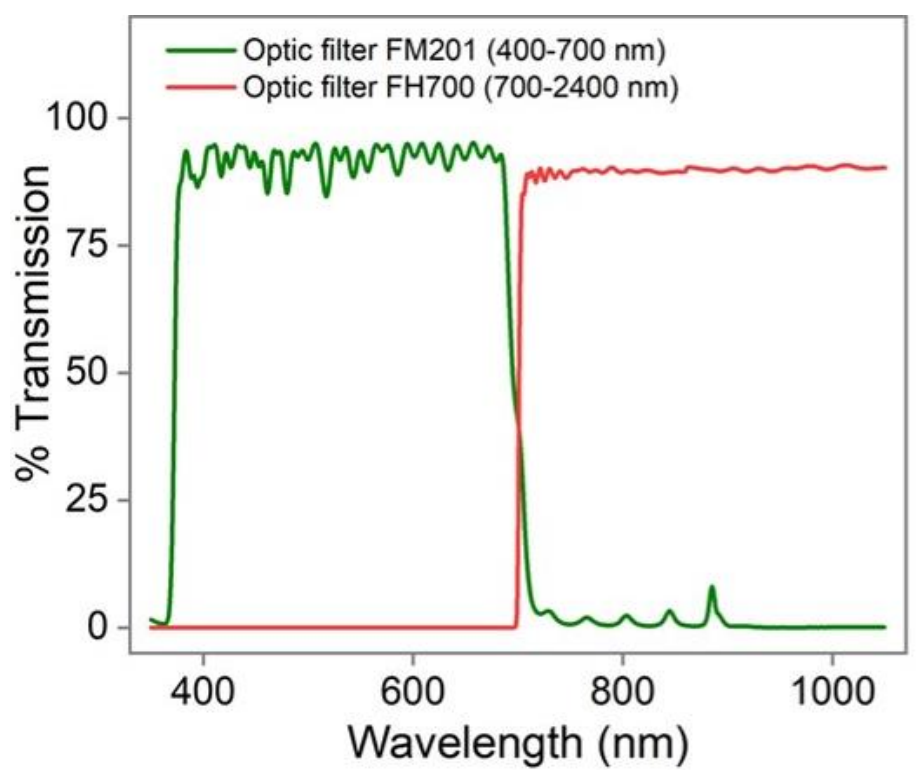

Figure S6. Transmission of the glass colored filter FM201 (green line) and FH700 (red line) used for the independent excitation of each plasmonic signature. 